\title{
Vom Leben der Geringsten
}

Michall PpelfFer

Universitat Pompeu i Fabra, Barcelona

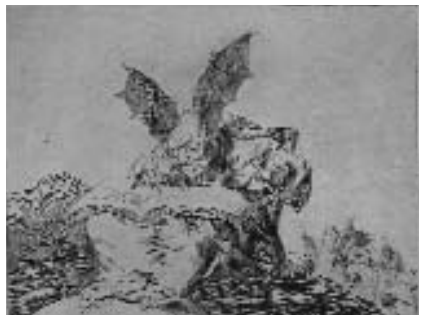

Omnes namque homines natura aequales sumus.

Gregor Der Grosse: Moralia in Iob

Wann immer in der Geschichte Europas das einfache Volk

mit den ursprünglichen Texten des Evangeliums in Berührung kam,

dann entbrannte sozialer Aufstand, denn es ist eine Botschaft

der menschlichen Gleichheit und Gerechtigkeit

und den Reichen feindlich gesinnt.

Golo Mann: Deutsche Geschichte des 19. und 20. Jahrhunderts

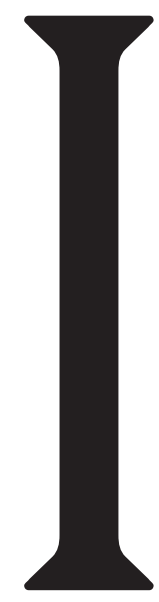

$\mathrm{n}$ seiner Rede zum Büchner-Preis hat Elias Canetti den Woyzeck als die „Entdeckung des Geringen « hervorgehoben. Mit dieser Entdeckung sei Georg Büchner „der vollkommenste Umsturz in der Literatur gelungen. «Und zwar laut Canetti deswegen, weil das Geringe bei Büchner „intakt« geblieben sei. Denn, so Canetti: „Der Dichter, der sich mit seinen Gefühlen spreizt, der das Geringe mit seinem Erbarmen öffentlich aufbläst, verunreinigt und zerstört es. «² (Canetti 1981: 239) Naturgemäß spricht Canetti hier auch von sich selbst und seinem Werk, von jenem Ohrenzeugen, der er war und der sich lauschend in die akustischen Masken der Sprecher seiner Umgebung hineinhörte, um deren lebendige Stimmen später zu Papier und auf der Bühne zu Gehör zu bringen. Das mimetische Abhören des Realen verbindet Canetti mit seinem Vorbild Büchner. Beide arbeiten mit Vorgefundenem, Abgehörten und collagieren es dann zu ihrer eigenen Sprachkunst. Damit gehen sie über das rein Dokumentarische hinaus, was heute andere verdienstvolle Autoren wie Günter Wallraff von Ganz unten oder Walter Kempowski mit seinem Echolot aus dem Leben der Geringen zu Tage förderten.

Allerdings, was Canetti als die „Entdeckung des Geringen « sogar kursiv hervorhebt, ist selbst wiederum ein nicht ganz genaues Zitat aus Büchners Erzählung Lenz, und dort aus dem sogenannten „Kunstgespräch «, der zentralen Passage, die uns einen der wenigen Einblicke in Büchners Vorstellungen von dem gibt, was sein Kunstprinzip um das Jahr 1835 gewesen sein könnte: „Man versuche es einmal und senke sich in das Leben des Geringsten und gebe es wieder, in den Zuckungen, den Andeutungen, dem ganzen feinen, kaum bemerkten Mienenspiel. «Dieses geringe Leben, ob schön oder hässlich, „sei das einzige Kriterium in Kunstsachen. Übrigens begegne es uns nur selten, in Shakespeare finden wir es und in den Volksliedern tönt es einem ganz, in Göthe manchmal entgegen. « Alles Übrige könne man ins Feuer werfen. Lenz alias Büchner kanzelt dann mit einem einzigen Satz all jene Idealisten seiner Zeit ab, die sich wohl auch noch zu Zeiten Canettis mit ihren Gefühlen und Erbarmen spreizen und öffentlich aufblasen wollen: Solche Leute (Schiller ist unter anderen gemeint) könnten nicht einmal „einen Hundsstall zeichnen. « Sie wollten „idealistische Gestalten « geben, er habe davon aber nur „Holzpuppen« gesehen. Kurzum: „Dieser Idealismus ist die schmählichste Verachtung der menschlichen Natur.« An diesen apodiktischen Satz schließt Büchners seine ins noch Unbekannte tastende, bereits zitierte, antiidealistische Forderung an die Literatur an: „Man versuche es einmal und senke sich in das Leben des Geringsten und gebe es wieder, in den Zuckungen, den Andeutungen, dem ganzen feinen, kaum bemerkten Mienenspiel. « (SW1: 234) Bewundernswert, in welcher Kürze dieser junge Medizinstudent sein künstlerisches Verfahren auf den Punkt bringt. Auch der Anatom Büchner scheint in dem Satz durch, der sein Skalpell an kaum sichtbaren Nervenbahnen (Zuckungen) von Fischen entlangzuführen weiß. Dennoch, es geht hier literarisch um ein Sich-Versenken in das Geringste, um Empathie oder, um mit Goethes Ideen zur Morphologie zu sprechen, um eine „zarte Empirie « mit dem Geringsten (bei Büchner im Superlativ, bei Canetti bescheidener zitiert). Schon ein Jahr vor der Arbeit am Lenz hatte er im Februar 1834 von einer vergleichbar „schändlichen « Menschenverachtung an die Familie geschrieben:

\begin{abstract}
„Es ist deren eine große Zahl, die im Besitze einer lächerlichen Äußerlichkeit, die man Bildung, oder eines toten Krams, den man Gelehrsamkeit heißt, die große Masse ihrer Brüder ihrem verachtenden Egoismus opfern. Der Aristocratismus ist die schändlichste Verachtung des heiligen Geistes im Menschen; gegen ihn kehre ich seine eigenen Waffen.«(SW2: 379)
\end{abstract}

Ein Jahr später, ab 1836, wird er sein literarisches Programm mit der Versuchsanordnung des Woyzeck ins Werk setzen, wird sich zeitweise in das Leben des armen Soldaten Franz Woyzeck und seiner Marie senken, um es in einem Drama wiederzugeben, mit dem erst viele Jahrzehnte später, letztlich im 20. Jahrhundert „der vollkommenste Umsturz in der Literatur « tatsächlich gelingen sollte. 


\section{Abstract}

Elias Canetti feierte den Woyzeck als die „Entdeckung des Geringen“ and damit als „den vollkommensten Umsturz in der Literatur". Der Artikel differenziert dieses treffende Urteil mit Büchners eigenen Aussagen und den Arbeiten der Büchner-Hermeneutik (Dedner, Deiters, Auerbach, Kurzke). Um die literarische Revolution des Woyzeck zu erklären, beleuchtet er die Begriffsgeschichte des Wortes ,Volk‘ zwischen französischer (La Bruyère, Voltaire, Balzac) und deutscher Belegung (Goethe, Herder, Freiligrath, Grimm), und unterstreicht Büchners Leistung, den ,unüberwindbaren Riss in der Gesellschaft ' kritisch auszuloten und in Kunst zu verwandeln.

Schlüsselwörter: Büchner, Woyzeck, Volk, Volkskultur

\section{Resumen}

Elias Canetti celebró el Woyzeck como el «descubrimiento de lo ínfimo» y con ello como «la revolución más completa en la literatura». El artículo matiza este certero juicio de la mano de los testimonios del propio Büchner y los trabajos hermenéuticos de Dedner, Deiters, Auerbach y Kurzke. Para ilustrar la revolución literaria del Woyzeck, recorre la historia del término 'pueblo' en sus acepciones francesa (La Bruyère, Voltaire, Balzac etc.) y alemana (Herder, Goethe, Freiligrath, Grimm) para resaltar el logro de Büchner de verter luz sobre el 'abismo insalvable en la sociedad' y convertirlo en arte.

Palabras clave: Büchner, Woyzeck, pueblo, cultura popular.

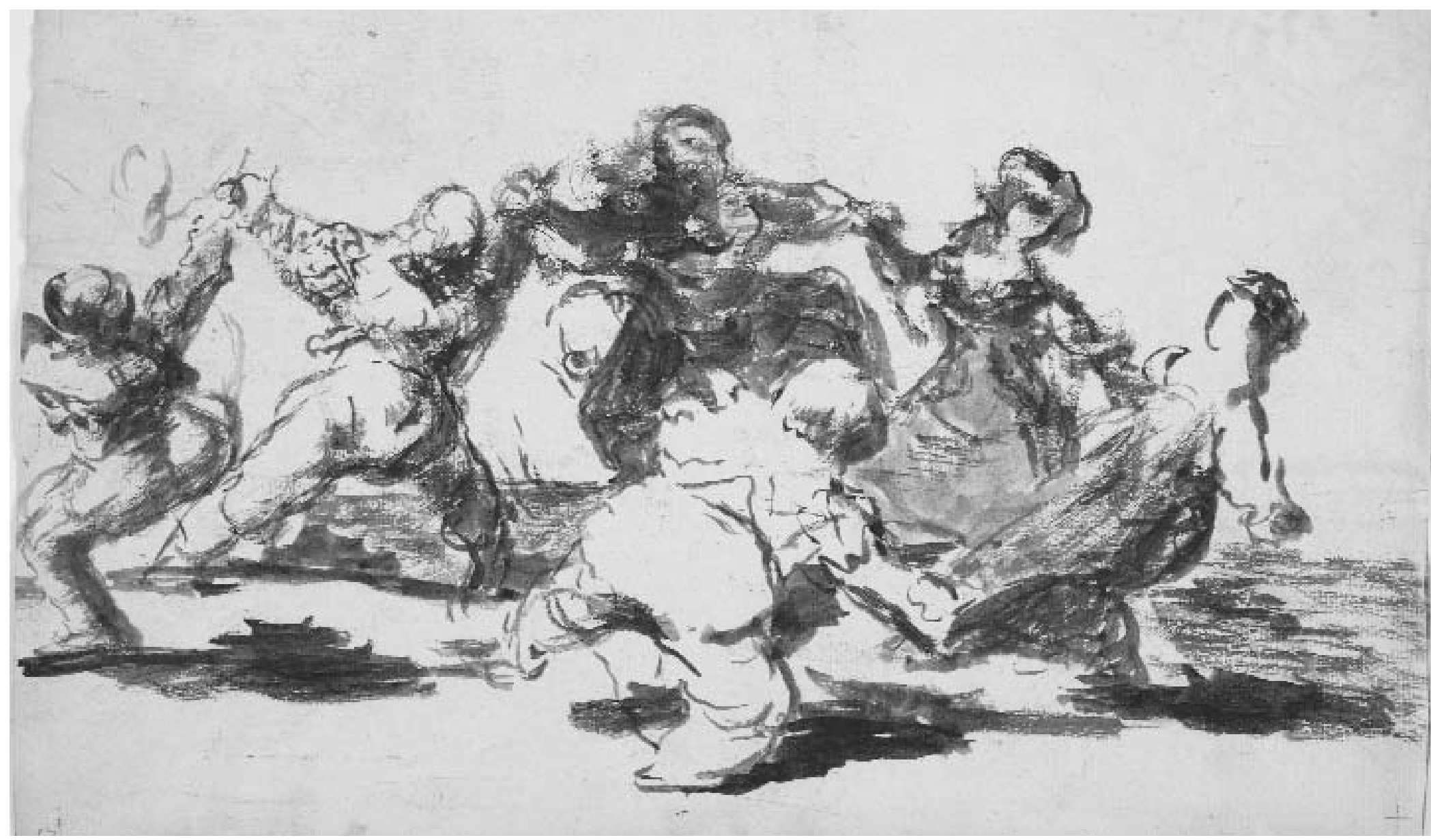

Historisch gesehen ist Büchners Umsturzversuch in der Literatur auch insofern ein einmaliges Unterfangen, als die Geringen oder das sogenannte „Volk « sich ja nicht selbst zur Sprache gebracht hat, weder literarisch noch politisch gibt es Selbstaussagen. Auch der privilegierte Student und Bürgersohn Georg Büchner ist keiner von denen da unten, sondern nimmt die Geringen notwendig von oben (, ,sich senken «) in den Blick. Nur war er sich im Unterschied zu vielen anderen Autoren dieses Unterschiedes höchst bewusst. An Karl Gutzkow schreibt er im Juni 1836: „Sie werden nie über den Riß zwischen der gebildeten und ungebildeten Gesellschaft hinauskommen. (SW2: 440) Diese Erfahrung des unüberwindlichen Risses in der Gesellschaft hatte er zwei Jahre zuvor als politisch motivierter Mitverfasser des Hessischen Landboten von 1834 auf schmerzliche Weise machen 
müssen, als nämlich die Wirkungslosigkeit seiner und Pfarrer Weidigs Flugschrift offenbar wurde. Wie bekannt händigten die ungebildeten Adressaten der Flugschrift ihre Exemplare brav an die Polizei aus (SW 2: 844; Buck 2000, 11). Nicht jeder Bauer gerät gleich zum Wilhelm Tell vom Schlage Schillers, den Büchner, wie gesagt, nicht sehr schätzte. „Mästen Sie die Bauern «, schreibt er im Jahr darauf bitter enttäuscht an Gutzkow, „und die Revolution bekommt die Apoplexie. Ein Hubn im Topf jedes Bauern macht den gallischen Hahn verenden. (SW2: 400)

\section{Die Entdeckung der Geringsten}

Warum aber kann Canetti von einer „Entdeckung « des Geringen sprechen, als ob dieses nicht doch seit eh und je vor der Haustür lebte? Und Büchner spricht von einem Versuch, den man machen solle, sich wie eine Expedition in unbekannte untere Zonen der Gesellschaft zu begeben. In der literarischen Tradition bekannt und beliebt war das Volk, waren die Geringen als Lachfiguren von Komödie und Satire. Masken, grobe Fratzen und Grimassen zeichnen sie aus, gerade das Gegenteil von jenem Leben, das Büchner mit seinem „feinen, kaum bemerkten Mienenspiel « an die Oberfläche heben will, jene Sensibilität und Wahrheit, die dem gemeinen Volk sozial und ästhetisch wegen der Ständeklausel keineswegs zustand. „Es sind die prosaischsten Menschen unter der Sonne; aber die Gefühlsader ist in fast allen Menschen gleich, nur ist die Hülle mehr oder weniger dicht, durch die sie brechen muß. Man muß nur Aug und Ohren dafür haben «, hieß es weiter im Lenz. (SW1: 234)

Es lohnt, sich die Widerstände ins Gedächtnis zu rufen, die Mitte der 30er Jahre des 19. Jahrhunderts zu überwinden waren, um einen so prosaischen Menschen, wie den gemeinen Mörder Franz Woyzeck zum Protagonisten eines Dramas zu machen. Lassen wir die überkommenen ständischen Spielregeln in Kunst und Theater beiseite und wenden uns einem weit wirksameren Vorurteil $\mathrm{zu}$, das das Volk im Allgemeinen geringschätzte, selbst und gerade in einem so bürgerlichen Unternehmen wie der Aufklärung.

Im Europa der Aufklärung, zumal in Frankreich, vertiefte die intellektuelle Elite ab dem Ende des 17. Jahrhunderts jenen "Riß zwischen der gebildeten und ungebildeten Gesellschaft ", zwischen sich und der Masse der Bevölkerung. Bemerkenswert ist diese Tradition insofern, als die französische Aufklärung das Landvolk aus der Perspektive des exotischen „Wilden « in der Neuen Welt zum verdorbenen Wilden im eigenen Land stigmatisiert oder „entfremdet «, um diesen Vorgang mit dem literaturtheoretischen Terminus der ostranenie von Viktor Šklovskij zu beschreiben: der „Verseltsamung « oder „Entfremdung «. ${ }^{4}$ Prominentes Beispiel ist La Bruyères verseltsamende Beschreibung der Bauern in Les caractères von 1689, ohne diese allerdings als seine eigenen Landsleute beim Namen zu nennen. ${ }^{5}$ Ein heute äußerst irritierender Text, der zu Beginn von wilden Tieren handelt, die dann aber doch über so etwas wie eine artikulierte Stimme verfügen und Zeile nach Zeile letztlich ein menschliches Antlitz zeigen, also tatsächlich als Menschen zu gelten haben - mit wenn auch verachtenswerten Verhaltensweisen. Man fühlt sich unwillkürlich erinnert an den Ausrufer der Bude in der Jahrmarktszene des Woyzeck, an „die viehische Ver-
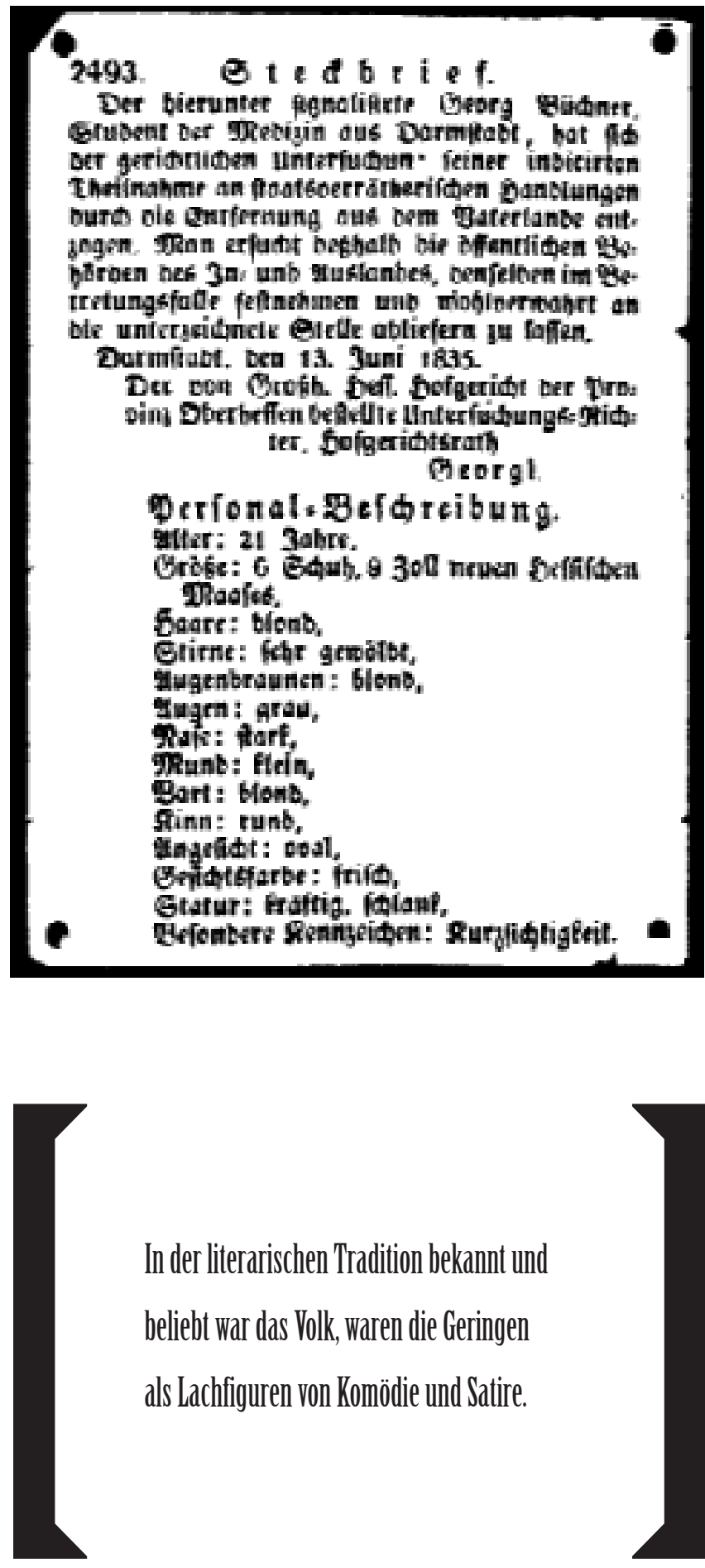

In der literarischen Tradition bekannt und

beliebt war das Volk, waren die Geringen

als Lachfiguren von Komödie und Satire.

nunft « und den Affen, der schon ein Soldat sei, „s'ist noch nit viel, unterst Stuf von menschliche Geschlecht! «(SW1: 150)

Voltaire schließt an La Bruyères Charakterisierung der Bauern in dem Kapitel Des sauvages seiner Philosophie de l'Histoire (1765) mit einer rhetorischen Frage an: Soll man unter Wilden also arme, hart schaffen müssende Bauerntölpel verstehen, dem Städter unverständliche Dialektsprecher, blind abhängig von Steuerschreibern, Teilnehmer an ihnen undurchsichtigen Zeremonien (Messe), wo ein verkleideter Mann (Priester) von ihnen 


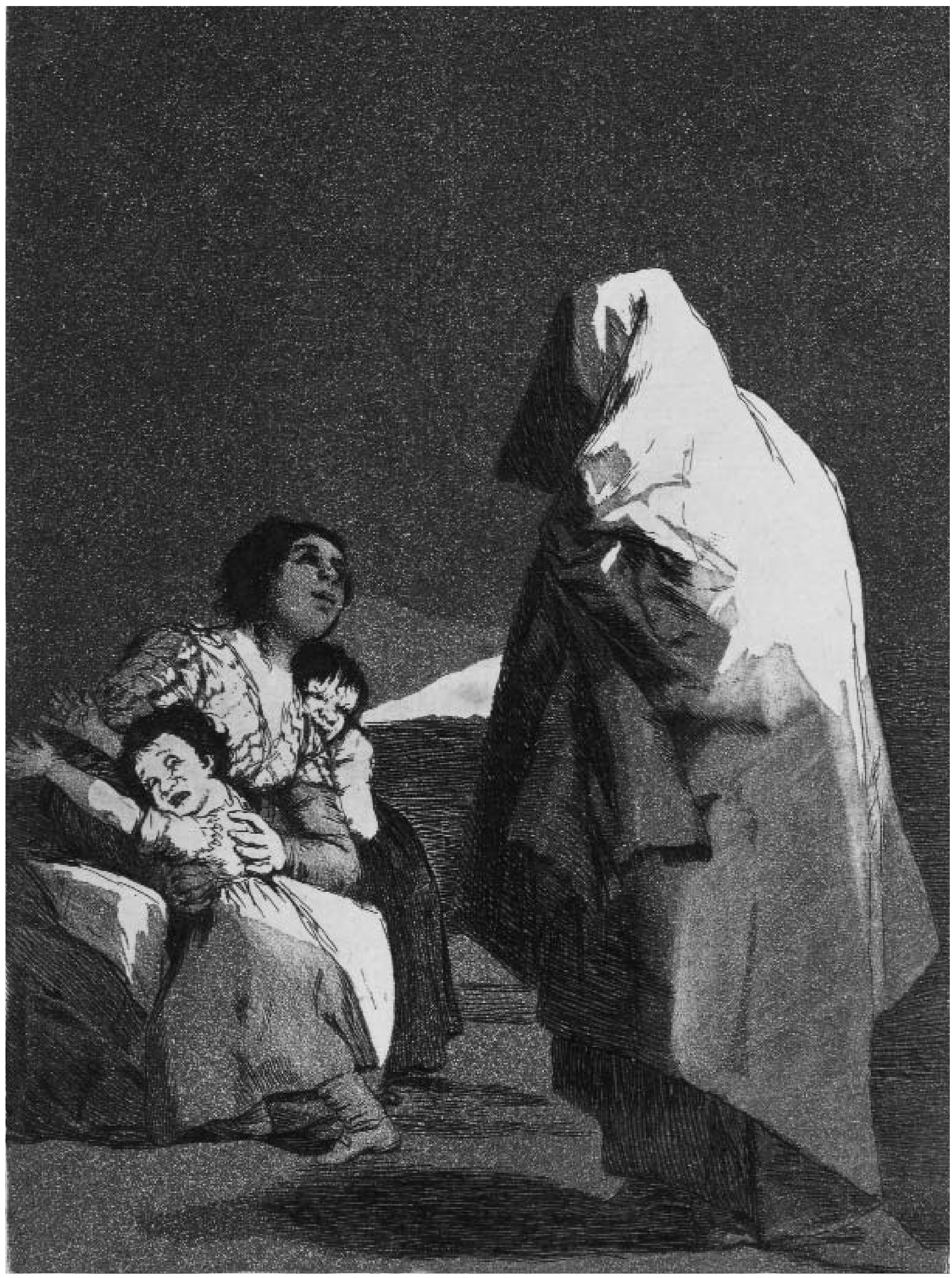


so wenig versteht wie sie von ihm, und die letztlich zum Schlag der Trommel für kargen Sold in fremden Ländern einander totschlagen? ${ }^{6}$ Wilde dieser Art, so die Antwort, bevölkern ganz Europa und nicht etwa die Neue Welt. Was „unseren « Bauerntölpeln, „unseren « Wilden fehle, so schließt Voltaire, sei die Idee der Freiheit. Der gemeine Mann als Exot bleibt in Frankreich Tradition. Fünf Jahre nach Büchners Tod ist es Eugène Sue, der das Muster exotischer Alterität auf die einheimischen Pariser Unterschichten anwendet. Seinem überaus erfolgreichen Sittenbild Les Mystères de Paris (1842/1843) stellt er reißerisch werbende Betrachtungen voran. Jedermann habe die Romane Coopers - „le Walter Scott américain « - gelesen, in denen die grausamen Sitten fern von der Zivilisation lebender „sauvages « geschildert würden. Sein Roman nun sei der Versuch, dem Leser eine andere Art Barbaren in Paris vorzuführen. Im Vertrauen auf die ästhetische Wirkung starker Kontraste animiert er den Leser zu einer Exkursion mitten unter die Eingeborenen dieser infernalischen Rasse, um auf dieser Entdeckungsreise (investigation) den Fuß auf die unterste Sprosse der sozialen Stufenleiter zu setzen (dernier échelon de l'échelle sociale). ${ }^{7}$ Es blieb den Brüdern Goncourt vorbehalten, 1865 in der programmatischen Einleitung zu Germinie Lacerteux die Frage zu stellen, ob denn zu Zeiten des allgemeinen Wahlrechts, der Demokratie und des Liberalismus nicht auch jene, die man die niederen Klassen nenne, ein Recht auf den Roman hätten. ${ }^{8}$ Erich Auerbach allerdings sieht das Projekt eines „ernsten Realismus « auch bei den Goncourts noch recht skeptisch: „[...] sie waren Sammler und Darsteller von Sinneseindrücken, und zwar von solchen, die einen Seltenheits- und Neuigkeitswert hatten; sie waren, von Berufs wegen, Entdecker oder Wiederentdecker von ästhetischen, insbesondere von morbid-ästhetischen Erfahrungen, die einem anspruchsvollen, des Gewohnten überdrüssigen Geschmack Genüge tun konnten. Von diesem Standpunkt reizte sie das niedere Volk. « (Auerbach 462) Im ernsten Realismus hingegen müsse das niedere Volk, so Auerbach, in all seinen Teilen als Gegenstand einbezogen werden. Genau dies sei es aber, was den ersten großen Realisten des Jahrhunderts (Stendhal, Balzac und auch Flaubert) noch fehle: bei ihnen erscheinen die tieferen Schichten des Volkes, ja das eigentliche Volk überhaupt, noch kaum; „und wo es erscheint, da wird es nicht aus seinen eigenen Voraussetzungen, in seinem eigenen Leben, sondern von oben her gesehen. « (Auerbach 462)

Noch im 20. Jahrhundert scheint dieser Exotismus sogar in der historischen Betrachtung armer Leute des 19. Jahrhunderts nachzuklingen. Gemeint ist der Fall des französischen "Woyzeck «, des aus bäuerlichem Milieu stammenden dreifachen Mörders Pierre Rivière mit seinem selbst verfassten, wenn gleich von der Justiz eingeforderten „mémoire « aus dem Jahre 1835: "Moi pierre Riviere, ayant egorgé ma mére, ma soeur et mon frére «. Seine Aufzeichnungen samt einem Dossier wurden 1973 von Michel Foucault und Mitarbeitern herausgegeben, doch enthielt man sich in strengster Ausübung der „archéologie du silence « jeglicher Deutung von Rivières Text, um den Diskurs des Ausgeschlossenen nicht durch den der Ausschließenden zu kontaminieren. Was bleibt, ist die Beschreibung des Mörders als eines „Menschen ohne Zivilisation «, „,ein Tier ohne Instinkt «, ,ein mythisches, ein monströ-
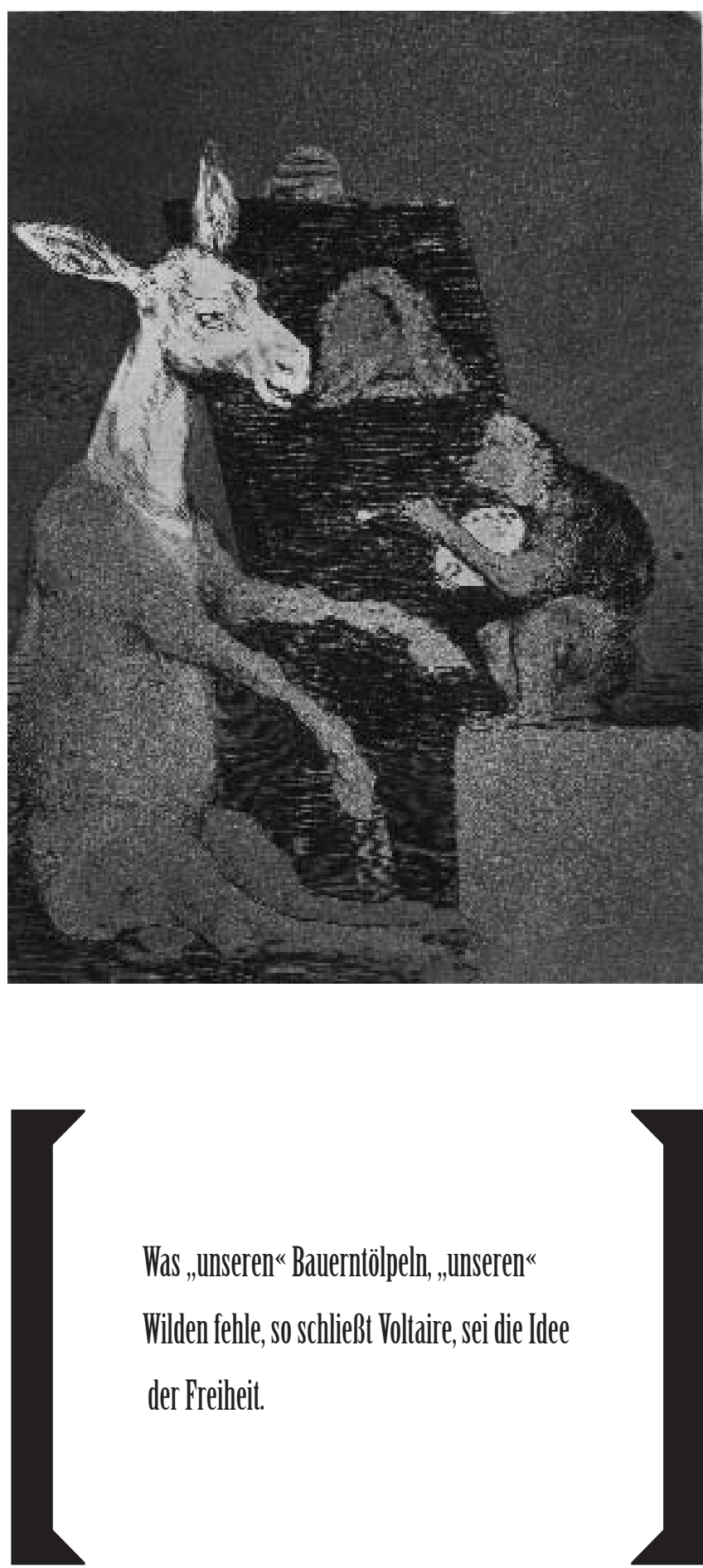

Was „unseren« Baulerntölpeln, „unseren“

Wilden fehle, so schließt Voltaire, sei die Idee

der Freiheit.

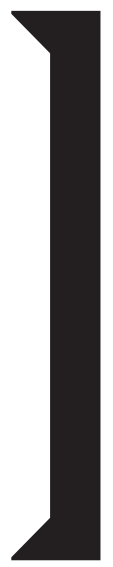

ses Wesen, dessen Definition unmöglich ist, weil es zu keiner nennbaren Gattung gehört «. 'Gegen diese diskurstheoretisch übertriebenen Vorbehalte Foucaults hatte Carlo Ginzburg seinerzeit bereits eingewandt, man sei „verzückt « gewesen „von einer absoluten Fremdheit, die in Wirklichkeit nur das Resultat der Weigerung zur Analyse und Interpretation « war. (Ginzburg 1979: 13)

Im deutschsprachigen Raum vollzog sich das, was Autoren der neueren Kulturgeschichte wie Peter Burke als Absonde- 
rung der Oberschicht vom „Volk« gegen Ende des 18. Jahrhunderts nannten, unter ganz anderen Voraussetzungen als in Frankreich, und es wäre eine vergleichende Studie wert, dem begriffsgeschichtlichen Unterschied von peuple und Volk nachzugehen. Um 1800 jedenfalls, der sogenannten Sattelzeit, wandelt sich der Begriff „Volk « in Deutschland von einem Sozialbegriff zu einem Zukunftsbegriff, wie Reinhart Koselleck nachweist. Als Sozialbegriff galt er „für die gemeinen Leute, den großen Haufen, die untersten Klassen der Besitzlosen und Nichtgebildeten, sowie - besonders pejorativ - für den Pöbel « (Koselleck 336) ${ }^{\text {Io }}$. Als Zukunftsbegriff verliert Volk seinen sozial beschreibenden Wert und weist auf eine zukünftig zu realisierende Einheit hin. Dieser semantische Umbruch lässt sich sehr deutlich an dem Eintrag „Volk« in Adelungs Wörterbuch von 1801 nachvollziehen. Der Artikel beginnt im Abschnitt e. mit dem Sozialbegriff der auf Arbeit angewiesenen unteren Schichten und definiert dann bis hinunter zum Pöbel: „Das Volk, das gemeine Volk, der große Haufe, gemeine Leute, die untersten Classen im Staat. Außer dem Oberherrn ist in einem Staate alles Volk, im weitesten Verstande. Da es denn in noch härterer Bedeutung oft von mehrern geringen Personen gebraucht wird. Es ist liederliches Volk, es sind liederliche Leute. « Nun kommt aber ein offenbarer Zusatz, der den aktuellen Sprachgebrauch der Jahrhundertwende reflektiert: „Einige neuere Schriftsteller haben dieses Wort in der Bedeutung des größten, aber untersten Theiles einer Nation oder bürgerlichen Gesellschaft wieder zu adeln gesucht, und es ist zu wünschen, daß solches allgemeinen Beyfall finde, indem es an einem Worte fehlet, den größten, aber unverdienter Weise verächtlichsten Theil des Staates mit einem edlen und unverfänglichen Worte zu bezeichnen. Romane für das Volk, Volksromanen, Volkslieder. « ${ }^{\text {II }}$ Dieser Prozess der Nobilitierung, der semantischen Aufwertung des Sozialbegriffs „Volk « ist das, was man seit Herders Sammeltätigkeit von Zeugnissen der Volkspoesie als die „Entdeckung des Volkes « bis in die Romantik hinein zu bezeichnen pflegt. Wobei man sich seit Herder tunlichst vom Pöbel absetzte: „Volk heißt nicht, der Pöbel auf den Gassen, der singt und dichtet niemals, sondern schreyt und verstümmelt. " ${ }^{12}$ Zugespitzt erscheint der Vorgang dann schließlich in den Liedzeilen Freiligraths um 1858: „Noch gestern, Brüder, wart ihr nur ein Haufen; ein Volk, o Brüder, seid ihr heut. «(nach Koselleck 147)

Erst vor diesem Hintergrund gewinnt Büchners antiidealistisches Programm auch die Kontur eines antiromantischen Programms. Wenn er vom Leben der Geringsten spricht, das selten in Shakespeare zu finden sei, aber einem manchmal bei Goethe und in Volksliedern „ganz « entgegentöne, dann sind damit sicher nicht jene Versionen der Volkslieder und Volksmärchen gemeint, die von den Romantikern zu einer Art Volkspoesie literarisch aufgearbeitet wurden. Auch gegen diese Art der Vereinnahmung von oben musste er seine prosaischen Figuren freihalten. Und entsprechenden Ärger verursachte er damit seinen Herausgebern. Es galt, sozusagen Jacob Grimms idealisierende Antwort auf die Frage, was ein Volk sei, von vornherein empirisch zu widerlegen. Auf der „Germanistenversammlung « in Frankfurt sollte Grimm 1846 behaupten: „ein volk ist der inbegriff von menschen, welche dieselbe sprache reden«. Gerade im Woyzeck aber machte Büchner
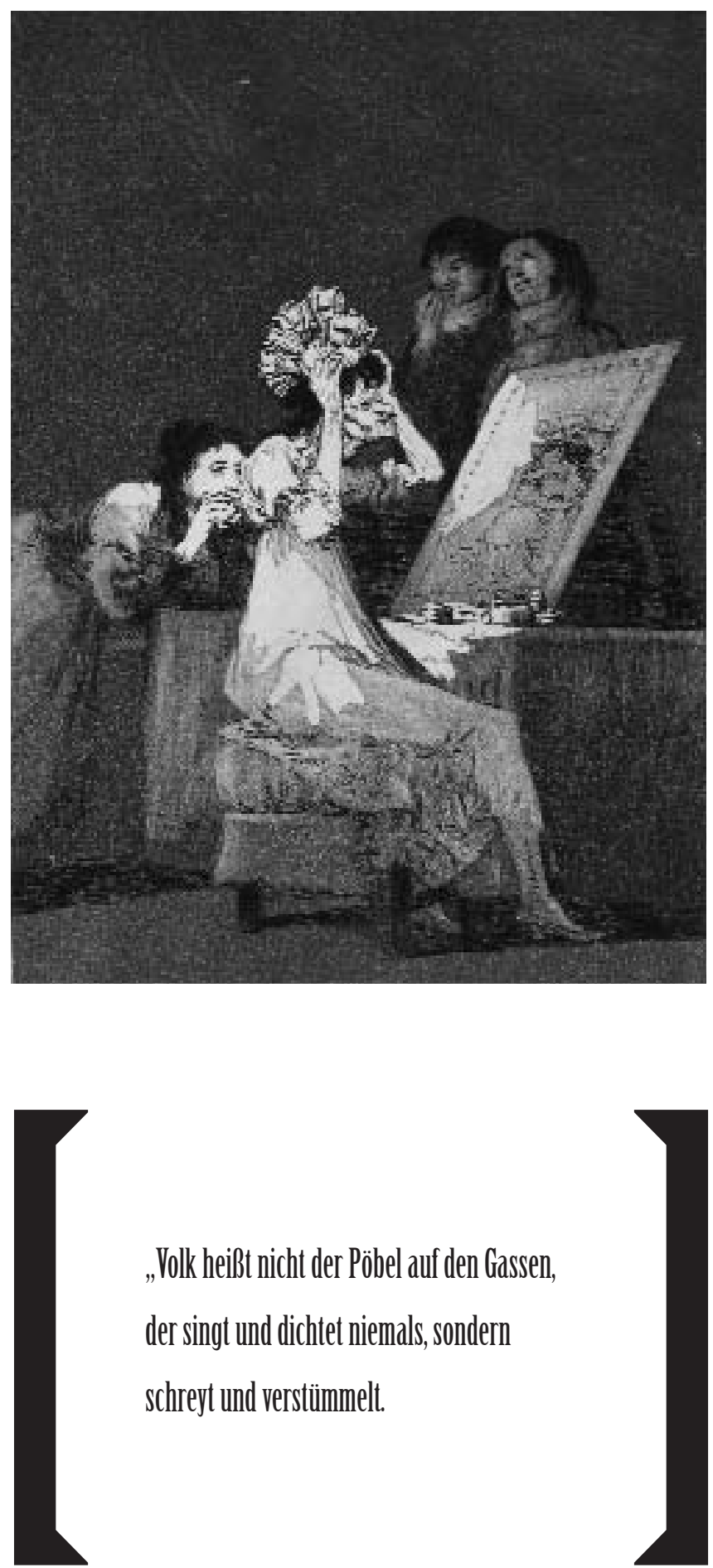

aber deutlich, dass dem nicht so sein kann. Bürger wie der Hauptmann und der Doktor sprechen eben nicht dieselbe Sprache wie der gemeine Mann Franz Woyzeck. Aber das ist, wie man heute sagen würde, soziolinguistisch gesehen und nicht national, oder empirisch und nicht idealistisch. Man könnte sich diese Divergenz auch höchst konkret vorstellen: In einem Dorf in Hessen-Darmstadt wird am Morgen heimlich der Hessische Landbote verteilt, und am Nachmittag erscheinen Märchen- und Sagensammler im Gefolge der Brüder Grimm, um nach alten Geschichten zu fra- 
gen. Wahrscheinlich werden die Bauern all diese verdächtigen, bebrillten Gelehrten zum Teufel geschickt haben. Es war übrigens niemand geringerer als Achim von Arnim, der 1813 wegen der „Unschuld der Volkssagen « warnend an Jacob Grimm geschrieben hatte: „So laß Dir denn sagen, weil Du zuweilen einer Behauptung zuliebe alle Gelehrsamkeit vergißt, daß die Hauptmasse von Volkssagen und -Liedern Zoten sind, das heißt ein Spott mit dem, was von der allgemeinen Meinung im Volke als sehr ernst betrachtet wird, insbesondere mit dem, was dem Familienkreise des einfacheren Lebens das Wichtigste ist, mit dem Ehestande und seinen Verhältnissen. ${ }^{\text {I4 }}$ Vor diesem Hintergrund lässt sich erahnen, dass der Woyzeck mit seinen Zoten, Hurereien und übers Kreuz pissenden Handwerksburschen oder dem Märchen der Großmutter zu Lebzeiten Büchners niemals unzensiert hätte publiziert werden können. Schon Dantons Tod hatte erhebliche Streichungen und Umformulierungen hinnehmen müssen. ${ }^{15}$ Die Stimme des Volkes, gar des Pöbels bekommen wir in der Literatur des 19. Jahrhunderts und auch anderer Jahrhunderte nicht zu hören. Und wenn, dann kam es schnell zum Skandal. Man erinnere sich nur an Peter Rühmkorfs Sammlung Über das Volksvermögen. Exkurse in den literarischen Untergrund. ${ }^{16}$ Büchners Zeitgenosse Auguste Blanqui brachte diesen Tatbestand im Proze $\beta$ der Volksfreunde zu Paris (gedruckt 1832 in Straßburg) mit aller Deutlichkeit zum Ausdruck:

„Das Volk schreibt keine Journale, schickt weder Adressen noch Bittschriften an die Kammern, dies wäre verlorene Zeit, ja, was noch mehr ist: Alle Stimmen, die in der politischen Sphäre einigen Widerklang finden, die Stimmen der Salons, der Handelshäuser, der Kaffeehäuser, kurz aller Orte, wo sich die öffentliche Meinung bildet: alle diese Stimmen sind die der Bevorrechteten; keine, nicht Eine gehört dem Volke an! Es ist stumm, es lebt sein Pflanzenleben, fern von diesen erhabenen Regionen; wo sein Schicksal abgekartet und bestimmt wird.« (zitiert nach Dedner 2007: 200f)

Blanquis Einwand entkommt allerdings nicht der Aporie seiner eigenen Einsicht: eine Stimme des Volkes ist auch er nicht, ebenso wenig wie Büchner. ${ }^{17}$ Noch als Revolutionär scheint er taub gegenüber dem, was er „von oben« gesehen zum sprachlosen „Pflanzenleben« des Volkes herabsetzt. Und mit einer gewissen Arroganz stilisiert er sich zum Botaniker oder gar Gärtner des stummen Volkes, das aber so stumm doch eigentlich nicht sein kann, denn es spricht ständig, nur hört ihm oben offenbar keiner so recht zu. An genau dieser Stelle, wo sich der unüberwindbare Riss in der Gesellschaft auftut, scheint mir Büchner mit seinem desillusionierten Scharfsinn die Lage der Geringsten zu erkennen, sie aufzunehmen und letztlich in Kunst zu verwandeln.

Im Woyzeck ist viel über die Voraussetzungen und das Eigenleben des Volkes zu erfahren, auch wenn es notwendig „von oben her gesehen « ist (Auerbach 464). Was Büchner als unüberwindbaren Riss erkannt hatte, ist zugleich eine Schnittstelle im doppelten Sinn des Trennenden und Verbindenden. Auch Erich Auerbach hatte im Konzept seiner Mimesis einen viel tieferen Schnitt im Sinn als nur den von höheren und tieferen Stilebenen, jenen, den die Botschaft des Evangeliums von der griechisch-römischen
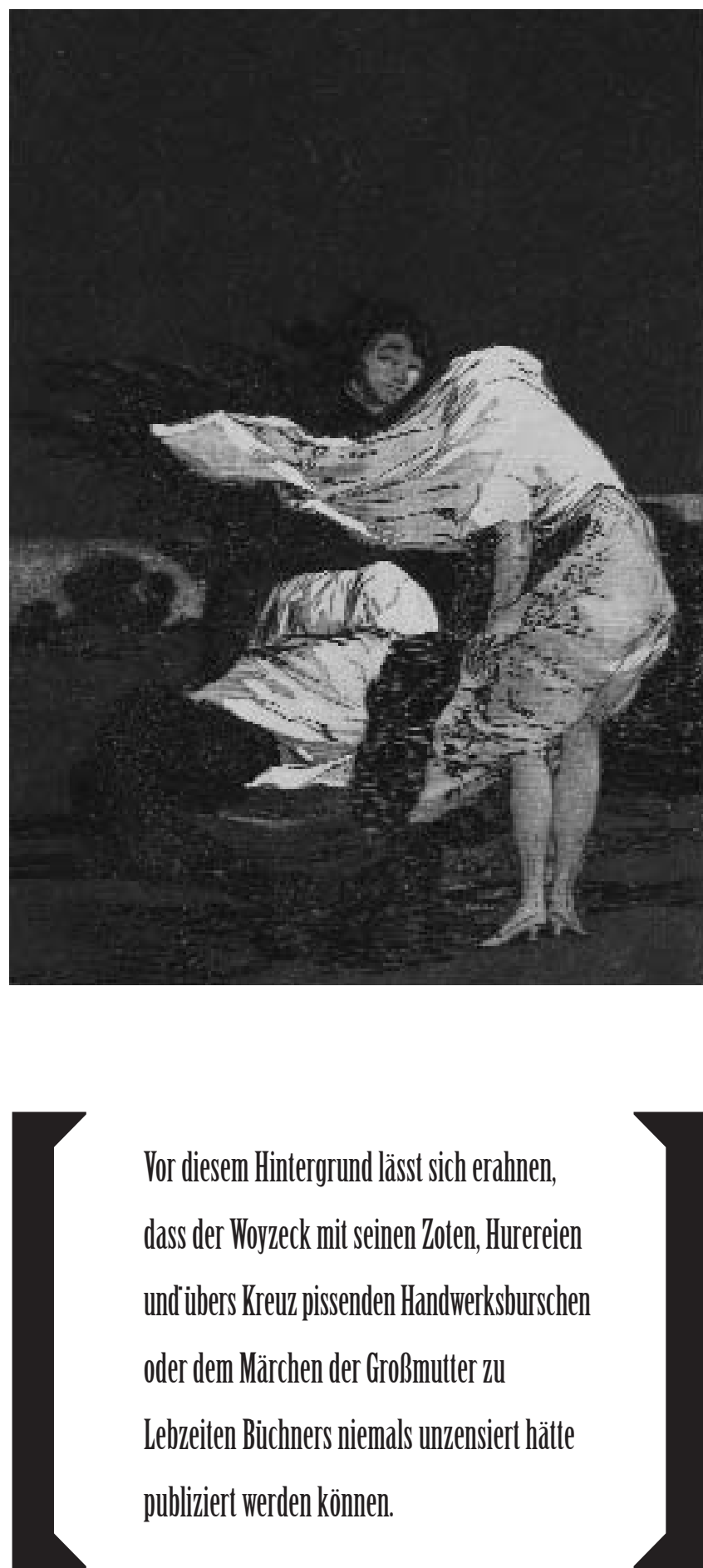

Vor diesem Hintergrund lässt sich erahnen, dass der Woyzeck mit seinen Zoten, Hurereien und übers Kreuz pissenden Handwerksburschen oder dem Märchen der Großmutter zu Lebzeiten Biuchners niemals unzensiert hätte publiziert werden können.

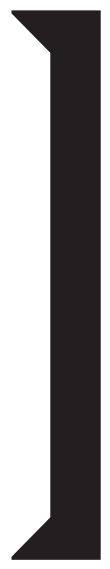

Antike trennt: den Siegeszug eines im Kuhstall geborenen Schreinersohnes gegen die Autoritäten der antiken Welt und deren Regeln in Kunst und Gesellschaft. Die scharfe Scheidung zwischen der Heiligen Schrift mit ihrer ursprünglichen Botschaft einerseits und der Institution Kirche samt ihrem dogmatischen Überbau andererseits findet sich in aller Deutlichkeit auch bei Büchner. Es ist der arme Woyzeck, der den Hauptmann mit wörtlichen Bibelzitaten aus seinem brüchigen Konzept bringt. Während dieser in seiner leerläufigen Manier „Moral« als ein „gutes Wort« definiert 
und Woyzeck vorwirft, er habe ein Kind „ohne den Segen der Kirche, wie unser hochehrwürdiger Herr Garnisonsprediger sagt, ohne den Segen der Kirche, es ist nicht von mir. «, kontert Woyzeck damit, dass der „liebe Gott« den „,armen Wurm« nicht darum ansehen wird, „ob das Amen der Kirche drüber gesagt ist, eh `er gemacht wurde. Der Herr sprach: Lasset die Kindlein zu mir kommen. «(SW1: 155) Allein dieser Passus des Disputs zeigt, wie treffgenau Büchner die Worte setzt. Woyzeck braucht nicht anzufügen, dass das Bibelzitat nicht von ihm sei, es sprach die Stimme des Herrn, während der Terminus „Herr« im Vorwurf des Hauptmanns nur aus der Anredeform für den Pfarrer sein geringes Gewicht bezieht. Und so hebelt Woyzeck wie ein listiger Markolf in der ganzen Szene die Scheinargumente seines Vorgesetzten aus.

Woyzecks eigenständiges Philosophieren dürfte Spuren dessen enthalten, was als nicht dokumentierter Volksglaube allenfalls in Inquisitionsakten oder Gerichtsgutachten zu finden ist, und dort immer in der kontaminierten Form der Abweichung, als aberratio mentalis oder Aberglaube behandelt wird. Carlo Ginzburg bezeichnet diese Quellen als eine Art „Schutzmauer « zum Ausschluss der Häresie, in die dennoch ab und zu eine Öffnung bricht:

„Durch die Diskrepanz zwischen den Fragen der Richter und den Antworten der Angeklagten hindurch - eine Diskre- panz die weder dem suggestiven Verhör noch der Folter zugeschrieben werden kann - leuchtet eine Tiefenschicht volkstümlicher Glaubensformen, die grundlegend autonom und unabhängig waren. «(Ginzburg 1983: 14)

Vielleicht würde Ginzburg inzwischen die „grundlegende « Autonomie dieser Glaubensformen relativieren, aber dennoch scheinen mir zum Beispiel Woyzecks Fragen nach der Lesbarkeit der doppelten Natur, „in was für Figur die Schwämme wachsen. Wer das lesen könnt. «(SW 1:158), auch auf jene Tiefenschichten zu verweisen, in denen sich christliche und pagane Religionsformen und -praktiken der Volkskultur vermengen. $^{\text {I8 }}$

Woyzeck wie auch Marie sind auf ihre Art auch gläubige Figuren, die im Evangelium eine Botschaft des Trostes und der Befreiung vernehmen, die aus ihrem Milieu stammt und ihre Sprache spricht. Ob sie die Botschaft auch annehmen, ist eine andere Frage. Man macht es sich zu leicht, vor allem in der früheren Büchnerforschung, wenn man dem Autor unterstellt, er sei permanent bereit, seine Bibelkenntnis „für die eigenen Aussageabsicht strategisch einzusetzen. «(SW2: 838) ${ }^{4}$ Denn, so lautet die noch immer offene und eigentlich müßige Frage: was wäre denn Büchners „Aussageabsicht«? Ein Stratege hat seine Werke sicher nicht geschrieben.

\section{Bibliographie:}

Auerbach, Erich (1959), Mimesis: Dargestellte Wirklichkeit in der abendländischen Literatur. Bern: A. Francke.

Brunner, Otto; Conze, Werner; Koselleck, Reinhart (Hrsg.) (1972 - 1992), Geschichtliche Grundbegriffe: Historisches Lexikon zur politisch-sozialen Sprache in Deutschland. Stuttgart: KlettCotta.

Büchner, Georg (1992), Sämtliche Werke, Briefe und Dokumente in zwei Bänden. Bd. 1: Dichtungen. Hgg.. von Henri Poschmann. Frankfurt am Main: Deutscher Klassiker Verlag.

Büchner, Georg (1999), Sämtliche Werke, Briefe und Dokumente in zwei Bänden. Bd. 2: Schriften, Briefe, Dokumente. Hgg. von Henri Poschmann. Frankfurt am Main: Deutscher Klassiker Verlag.

Buck, Theo (2000), „, Riß in der Schöpfung «. Büchner-Studien II. Aachen: Rimbaud.

Burke, Peter (1981), Helden, Schurken und Narren. Europäische Volkskultur in der frühen Neuzeit. Stuttgart: Klett-Cotta.

Canetti, Elias (1981), Büchner, in Das Gewissen der Worte. Essays. Frankfurt am Main: Fischer Verlag.

Dedner, Burghard (2007), Erläuterungen und Dokumente. Georg Büchner: Woyzeck. Stuttgart: Reclam.

Deiters, Franz-Josef (2006), Auf dem Schauplatz des ,Volkes «. Strategien der Selbstzuschreibung intellektueller Identität von Herder bis Büchner und darüber hinaus. Freiburg: Rombach

Ginzburg, Carlo (1996), Der Käse und die Würmer. Die Welt eines Müllers um 1600. Frankfurt am Main: Syndikat.

Ginzburg, Carlo (1996), Making Things Strange: The Prehistory of a Literary Device, Representations, No. 56/1996, S. 8-28.

Kurzke, Hermann (2013): Georg Büchner. Geschichte eines Genies. München: C. H. Beck.

Rühmkorf, Peter (1967), Über das Volksvermögen. Exkurse in den literarischen Untergrund. Reinbek: Rowohlt.

\section{Anmerkungen}

1 Diese Arbeit ist eine stark abgewandelte Fassung meines Artikels: „Woyzeck und Félicité. Arme Leut bei Büchner und Flaubert«. In: Michael Pfeiffer, Teresa Vinardell Puig, Anna Montané Forasté(Hg.): Was mich wirklich interessiert. Homenatge a Jordi Jané. Edició Fòrum 6, Barcelona 2012, Documenta Universitaria. S. 241-254.

2 Kursive Hervorhebungen im Original.

3 Unter dieser Sigle, sowie Band und Seitenzahl wird im Folgenden die Büchner-Ausgabe Sämtliche Werke, Briefe und Dokumente in zwei Bänden von H. Poschmann zitiert.

4 Vergl. hierzu Carlo Ginzburg: Making Things Strange. The Prehistory of a Literary Device. (Ginzburg 1996) Die folgenden Zitate sind diesem Aufsatz entnommen.

5 L'on voit certains animaux farouches, des mâles et des femelles, répandus par la campagne, noirs, livides et tout brûlés de soleil, attaches a la terre qu'ils fouillent et qu'ils remouent avec une opiniâtreté invincible; ils ont comme une voix articulée, et quand ils se lèvent sur leurs pieds, ils montrent une face humaine, et en effet ils sont des hommes; (Ginzburg 1996: 25)

6 Il y a de ces sauvages-là dans toute l'Europe. Il faut convenir surtout que les peuples du $\mathrm{Ca}$ nada et les Cafres, qu'il nous a plu d'appeler sauvages, sont infiniment supérieurs aux nôtres. [...] Les peuplades d'Amérique et d'Afrique sont libres, et nos sauvages n'ont pas même d'idée de la liberté. (Ginzburg 1996: 25)

7 Zitiert nach der digitalen Ausgabe: http://www.gutenberg.org/files/18921/18921-h/18921-h.htm. (Stand: 2/2/2013)

8 Zitiert nach E. Auerbach: Mimesis, (Auerbach 460)

9 Zitiert nach C. Ginzburg im Vorwort seines Klassikers Der Käse und die Würmer. Die Welt eines Müllers um 1600, (Ginzburg 1979: 13)

10 Unter dieser Sigle im Folgenden der Artikel ,Volk, Nation, Nationalismus, Masse" von R. Koselleck in Conze et. al.: Geschichtliche Grundbegriffe, Bd. 7.
11 Adelung: Grammatisch-kritisches Wörterbuch: «Volk «. 1801, Bd. 4, 1225. Zitiert nach Digitale Bibliothek, Bd. 40.

12 Herder, J. G.: Volkslieder. Nebst untermischten andern Stücken. Zweiter Theil. Leipzig: 1779, S. 20. Zitiert nach: http://www.unidue.de/lyriktheorie/texte/1779_herder.html\#fuss14g (Stand: 2. 2. 2013)

13 Vergl. hierzu auch Deiters 2006, S. 22-25.

14 Zitiert nach: Pikulik, Lothar: „Die sogenannte Heidelberger Romantik. Tendenzen, Grenzen, Widersprüche. Mit einem Epilog über das Nachleben der Romantik heute ". In: Heidelberg im säkularen Umbruch. Traditionsbewußtsein und Kulturpolitik um 1800. Hrsg. von Friedrich Strack. Stuttgart 1987. S. 190-215, hier: S. $198 \mathrm{f}$

15 Vergleiche zur Textgrundlage und Textgestaltung des Danton H. Poschmann in (SW1: 426ff).

16 Rühmkorf, P. (1967): Über das Volksvermögen. Exkurse in den literarischen Untergrund. Reinbek: Rowohlt.

17 Hierzu W. Conze in dem Artikel „Proletariat « bezogen auf den Pöbel: „Stets wurde dabei von aussen oder oben gewertet. Aussagen über „Pöbel « sind daher nie Selbstaussagen gewesen und konnten auch dann nicht zu solchen werden." In: Geschichtliche Grundbegriffe: Historisches Lexikon zurpolitisch-sozialen Sprache in Deutschland. Bd. 5, S. 29.

18 Einiges dazu findet sich wiederum im Lenz, wenn Oberlin und Lenz in die abgelegenen Dörfer des Steintales ziehen (SW1: 228-232)

19 Insofern schließe ich mich dem Grundtenor von Hermann Kurzkes (2013) Interpretation der Religiosität bei Büchner an. Es wäre aber präziser herauszuarbeiten, wo diese im Lenz und Woyzeck sich auch mit Einsprengseln aus dem sogenannten Volksglauben vermischt. 